\title{
Systematic echocardiographic assessment of aortic regurgitation- what should the surgeon know for aortic valve repair?
}

\author{
Alain Berrebi, Jean-Luc Monin, Emmanuel Lansac \\ Department of Cardiac Pathology, Institut Mutualiste Montsouris, Paris, France \\ Correspondence to: Alain Berrebi. Department of Cardiac Pathology, Institut Mutualiste Montsouris, 42 Boulevard Jourdan, 75014 Paris, France. \\ Email: alain.berrebi@imm.fr.
}

\begin{abstract}
Aortic valve (AV) repair is the preferred surgical treatment in young patients with aortic regurgitation (AR) and/or proximal aorta aneurysm, as noted in the recent European Society of Cardiology (ESC) guidelines. However, this surgical option is still underused in clinical practice. This emphasizes the need to build a heart team dedicated to AV repair with expert surgeons and echocardiographers. Surgical techniques are now standardized in their approaches to enhance the reproducibility and expansion of AV repair. The objective of this keynote is to also demonstrate the need for a standardized pre-pump intra-operative echocardiography protocol to fulfill surgeon's needs in providing a road map and predicting techniques to be used for an effective and durable repair.
\end{abstract}

Keywords: Intra-operative echo protocol; 3D echocardiography; aortic valve repair (AV repair); aortic regurgitation (AR); heart team

Submitted May 06, 2019. Accepted for publication May 13, 2019.

doi: $10.21037 /$ acs.2019.05.15

View this article at: http://dx.doi.org/10.21037/acs.2019.05.15

\section{Introduction: a need for standardization in a heart team approach}

Aortic valve (AV) repair is currently considered as a reference, a class I indication (level C) for the surgical treatment of aortic regurgitation (AR) in the new European Society of Cardiology (ESC) guidelines (1):

* "In patients with severe AR: heart team discussion is recommended in selected patients (patients with pliable non-calcified tricuspid or bicuspid valves who have a type I (enlargement of the aortic root with normal cusp motion) or type II (cusp prolapse) mechanism of AR in whom AV repair may be a feasible alternative to valve replacement";

* "In patients with aortic root or tubular ascending aortic aneurysm [for clinical decision making, dimensions of the aorta should be confirmed by electrocardiogram (ECG)-gated CT measurement] irrespective of the severity of AR: AV repair, using the reimplantation or remodelling with aortic annuloplasty technique, is recommended in young patients with aortic root dilation and tricuspid AVs, when performed by experienced surgeons".

However, according to the results from the Euro Heart Survey on Valvular Heart Disease (2), patients with tricuspid or bicuspid dystrophic AR, which represents the most common etiology in Western countries (two-thirds of AR cases), AV repair is still underused, performed in only $1.7 \%$ of cases. Facing these unmet needs, we have to promote AV repair to expand its indications in current practice. It emphasizes the need to build a heart team dedicated to AV repair with expert surgeons and echocardiographists. Surgical techniques are now standardized in their approaches to enhance the reproducibility and dissemination of AV repair (3). The objective of this keynote is to also demonstrate the need for a standardized pre-pump intra-operative echocardiography protocol to fulfill surgeon's needs in order to provide a road map and predict techniques to be used for an effective and durable repair. We will not cover the global role of echo in $\mathrm{AV}$ repair already well described by excellent reviews in the literature $(4,5)$. 
Tricuspid valve

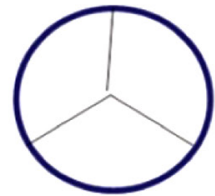

Commissures $120^{\circ}$
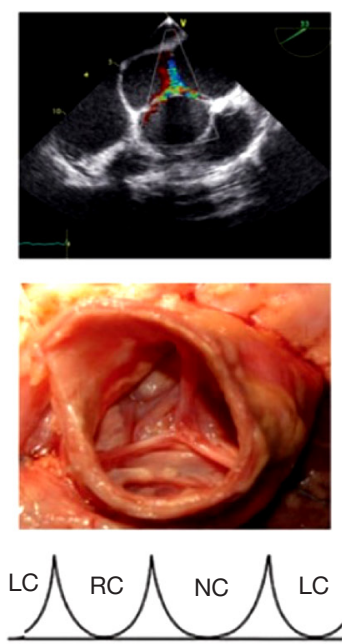

Bicuspid valve

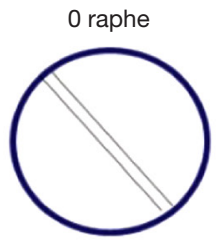

Commissures $180^{\circ}$
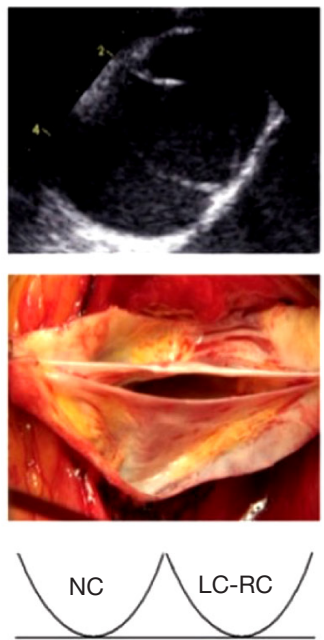

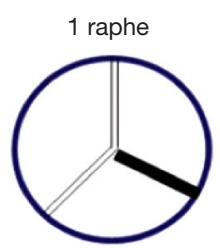

Commissures $120^{\circ}-180^{\circ}$
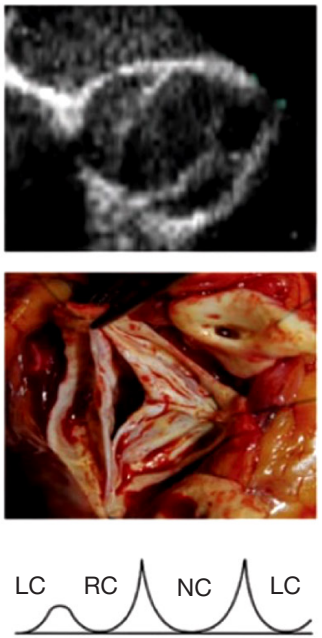

Unicuspid valve

2 raphes
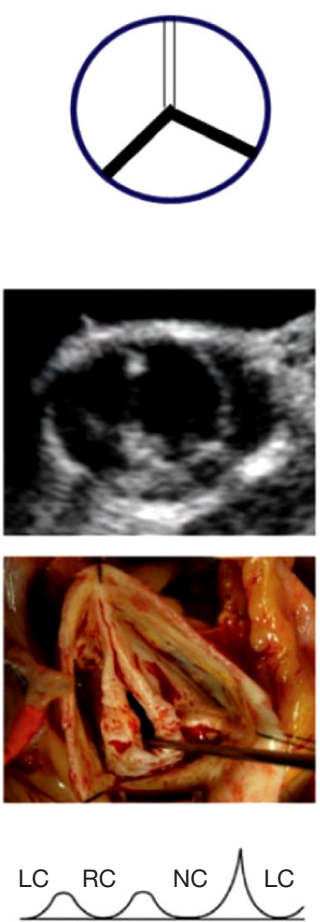

Figure 1 Valve type including commissural orientation and variation of aortic annulus morphology in relation to the leaflet insertion line. LC, left coronary; NC, noncoronary; RC, right coronary.

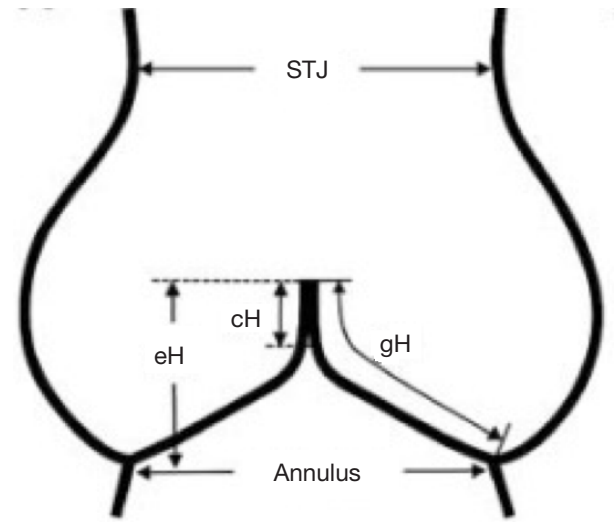

Figure 2 Definition of coaptation height $(\mathrm{cH})$, effective height $(\mathrm{eH})$ and geometric height $(\mathrm{gH})$ of aortic valve cusps. STJ, sinotubular junction.

\section{Terminology of AV repair measurements: a plea for a common language}

The first challenge of echocardiographist is to clearly understand surgeon's needs and the second is to create and adapt a tailored echo protocol which meets surgeon's needs.
Understanding definitions of specific terms commonly used in AV repair surgery to adapt corresponding echo measurements is the very first step to build an echo protocol in a heart team approach with a common language (Figures 1 and 2).

Valve type is defined by the number of cusps, which is based on the number of functional commissures (ordered by incidence):

(I) A tricuspid valve has three fully developed commissures;

(II) A bicuspid aortic valve (BAV) has two fully developed commissures and 0 or one raphe on the fused cusp, also classified as bicuspid type 0 or type I (6). A fused cusp is named according to the type of fusion, for example, R-L represents the most common type of right-left fusion;

(III) A unicuspid AV has one fully developed commissure and two raphes and should not be classified as bicuspid type II;

(IV) A quadricuspid valve has four commissures, one of which is often underdeveloped. 


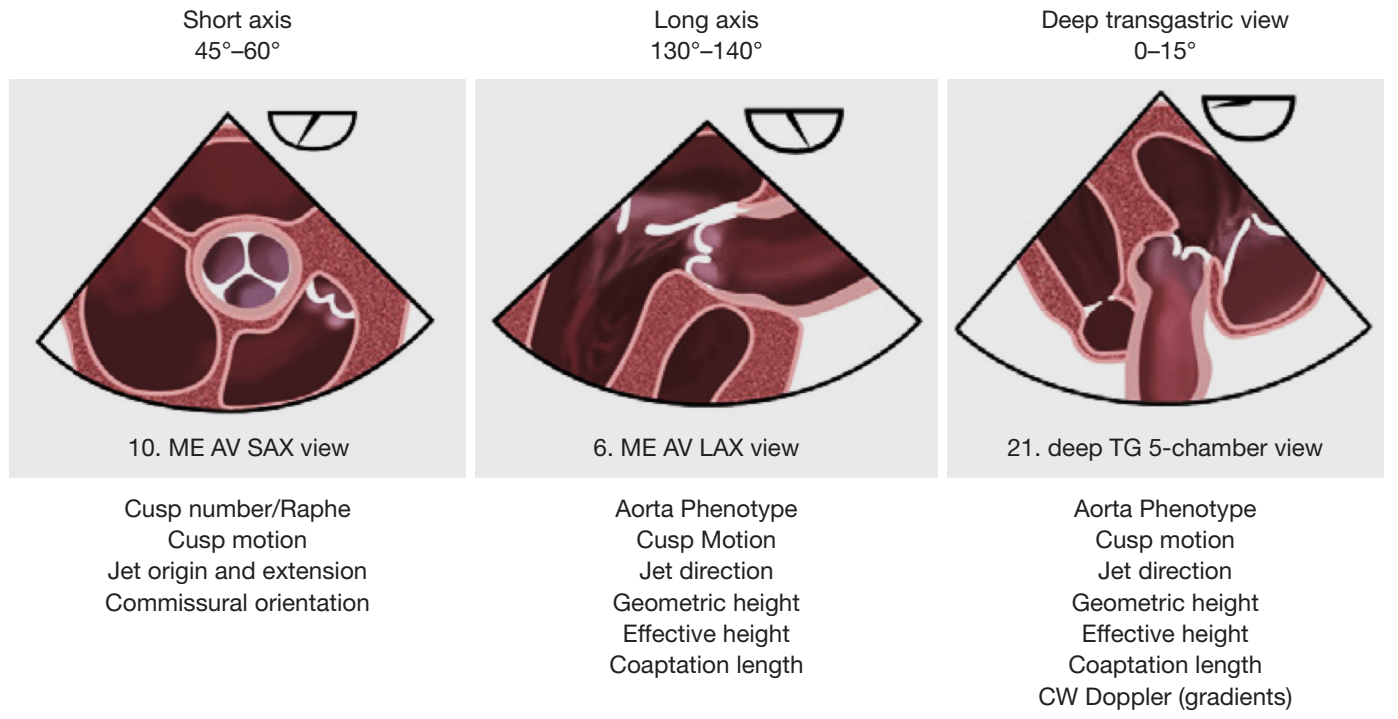

Figure 3 Echo views and measurements for AV repair. AV, aortic valve.

A raphe of the fused or conjoined cusp, also called a nonfunctional commissure (underdeveloped commissure with a hypoplastic interleaflet triangle), is the area of fusion between two abnormally developed aortic cusps. The raphe is characterized by a certain length of fusion between the two cusps and a certain height of insertion on the aortic root wall. The presence of one or two raphes, even if incomplete, make the valves anatomically bicuspid or unicuspid.

Commissure orientation is defined as the angle formed by the lines joining the commissures to the central axis of the valve. The angle measured is the one on the non-fused cusp side. It varies between $120^{\circ}$ (tricuspid configuration) and $180^{\circ}$ (bicuspid symmetrical configuration).

The aortic annulus is defined as the plane passing through the nadir of the aortic cusps that can be measured either using echocardiography to obtain the long axis view or by direct intubation intraoperatively.

The effective height $(\mathrm{eH})$ is the orthogonal distance from the annulus to the middle of the free margin of the cusp. The eH can be measured by echocardiography and intraoperatively with a dedicated caliper. The normal eH in the adult population is close to $9 \mathrm{~mm}(7)$.

The geometric height $(\mathrm{gH})$, also called the cusp height, is defined as the distance between the cusp nadir and the middle of the free margin. Intraoperatively, the $\mathrm{gH}$ is measured with a straight ruler along the aortic side of the cusp by applying gentle traction on the free margin to straighten the cusp tissue along the ruler. In adults, the cusp is considered retracted when the $\mathrm{gH}$ is $16 \mathrm{~mm}$ or less in trileaflet aortic valves (TAVs) and $19 \mathrm{~mm}$ or less in the bicuspid non-fused aortic cusp (8).

The coaptation height $(\mathrm{cH})$, also called the coaptation length (cL), is defined as the distance of cusp apposition in diastole. It can be measured echocardiographically on the long axis view of the $\mathrm{AV}$. The normal range is $4-5 \mathrm{~mm}$.

\section{TEE views and tools: the triumph of 3D approach}

The three basic 2D TEE views permit to obtain the measurements useful for surgeons (Figure 3).

* The mid-esophageal short axis view $\left(45^{\circ}\right.$ to $\left.60^{\circ}\right)$;

* The mid-esophageal long axis view $\left(130^{\circ}\right.$ to $\left.140^{\circ}\right)$;

- The deep transgastric view $\left(0-15^{\circ}\right)$.

In the short axis view, one can assess the number of aortic cusps, the presence/absence of one or several raphes, cusp motion, the origin and extension of the AR jet as well as the commissure orientation. The long axis view serves for the definition of the aortic phenotype, the assessment of cusp motion and jet direction, the measurement of the $\mathrm{gH}, \mathrm{eH}$ and coaptation length. The deep transgastric view is suitable for the assessment of the aortic phenotype, cusp motion, jet direction, geometric and eH, coaptation length as well as for the measurement of the $\mathrm{AV}$ pressure gradients by continuous wave Doppler (CWD) post AV repair.

However the 3D approach including all modalities (X Plane, 3D Live, 3D Zoom, Full volume, Multi Planar Reconstruction live with Multiview or post processing with 

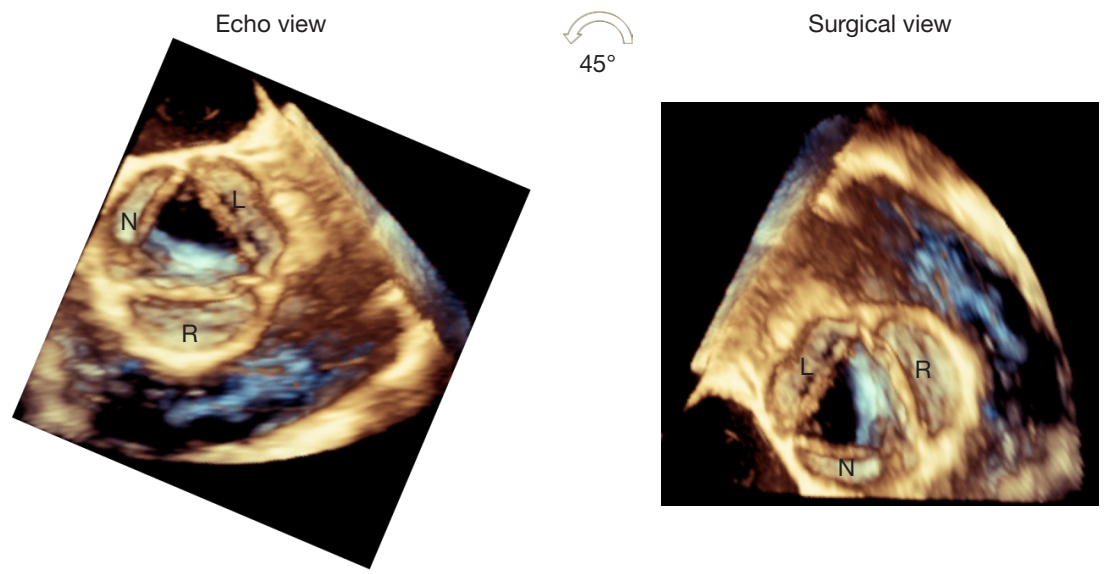

Figure 4 Surgical view only obtained with 3D echo (short axis).

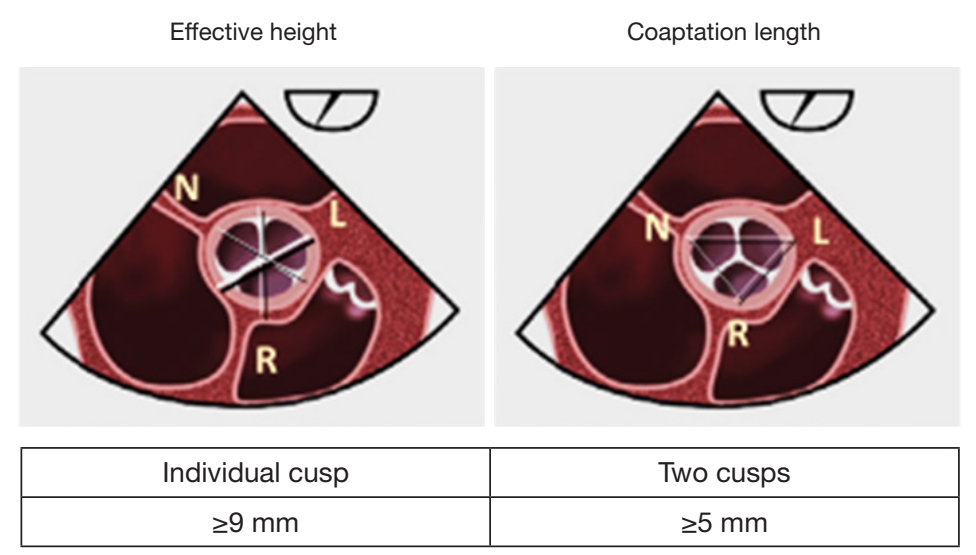

Figure 5 Cutting planes for effective height vs. coaptation height used with 3D tools.

Q Lab) is the best approach to fulfill the surgeon's needs (9). The superiority of $3 \mathrm{D}$ is illustrated by several advantages:

* It is important to bear in mind that the short axis TEE view of the AV (with the right coronary cusp displayed at the bottom, the noncoronary cusp to the left and the left cusp to the right) is not a true surgical view. The true surgical view is obtained by a $45^{\circ}$ counterclockwise rotation of the $3 \mathrm{D}$ short axis view of the $A V$, which brings the right coronary cusp on the right side of the screen, the left cusp on the left side and the noncoronary cusp at the bottom (Figure 4).

* It represents the sole method to individualize each cusp (tricuspid valve) or non-fused cusp (bicuspid valve). This advantage is crucial for measurements of $\mathrm{eH}$ and $\mathrm{gH}$. The eH is an individual measurement, which needs to be performed separately, for each of the three cusps. It is defined as the perpendicular between the $\mathrm{AV}$ annulus and the highest point of the free margin of each cusp, with a normal value $\geq 9 \mathrm{~mm}$. The coaptation length, on the other hand, is performed between each two cusps and represents the distance of cusp apposition with a normal value $\geq 5 \mathrm{~mm}$ (Figure 5).

$\mathrm{eH}$ measurement may be challenging, requiring a $3 \mathrm{D}$ acquisition of the AV. Starting with a $2 \mathrm{D}$ short axis view of the $\mathrm{AV}$, the $\mathrm{X}$ Plane tool can be employed to transect the middle of the right coronary cusp (located at the bottom), thus creating a long axis view of the AV which enables the measurement of the eH of the right cusp (Figure 6).

For the other two cusps, the $\mathrm{eH}$ is measured using a $3 \mathrm{D}$ acquisition (3D Zoom or Full volume of the AV and root), using the multiplanar reconstruction (MPR) to transect successively the middle part of each of the two cusps (the $\mathrm{X}$ Plane tool is not reliable in this situation as it intersects the 


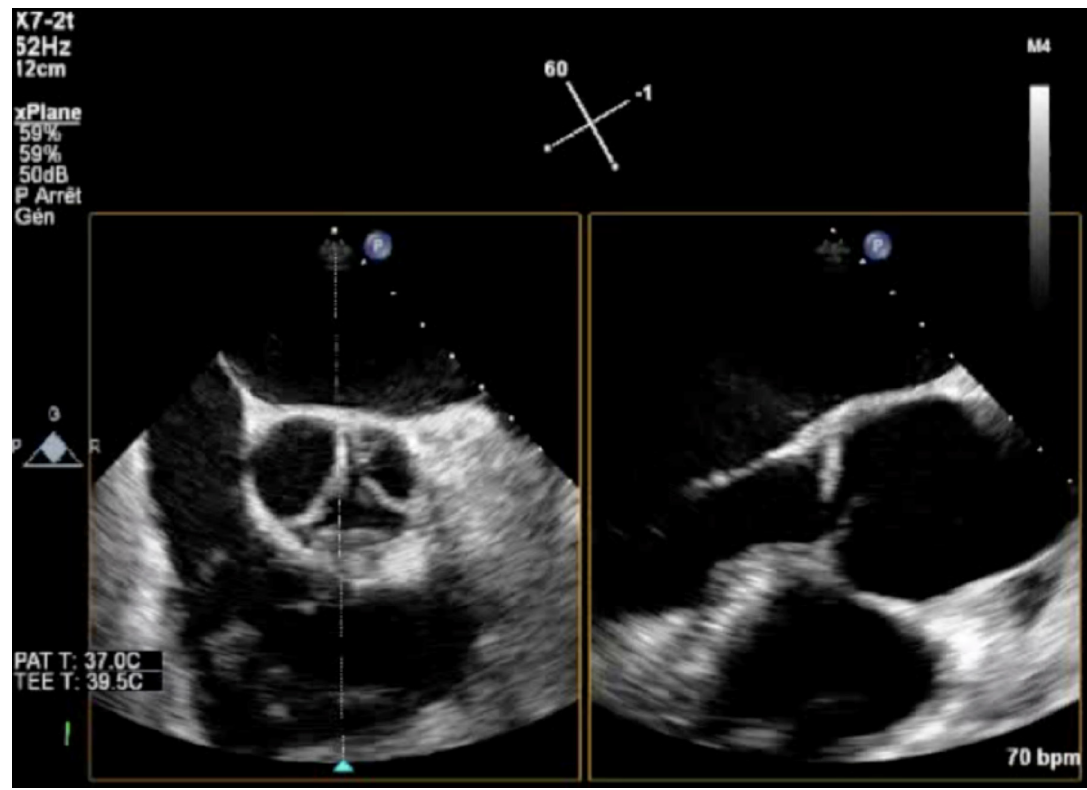

Figure 6 Effective height measurement of right cusp with X Plane tool.

NC

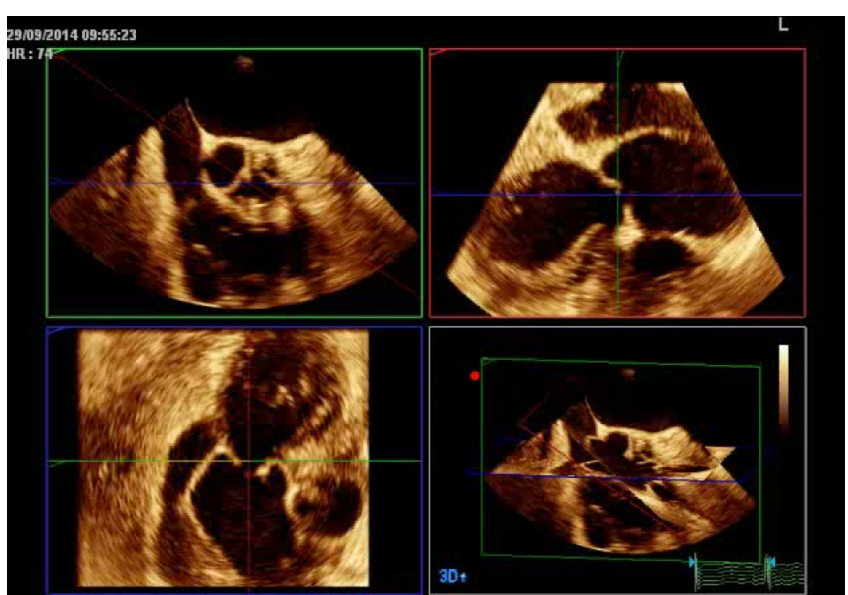

Figure 7 MPR alignment with a post-processing tool (Q Lab).

left and noncoronary cusps in an oblique fashion). It was done in a post-processing way with MPR software (Q Lab) (Figure 7).

Nonetheless, latest echo tools provide the possibility of live MPR alignment (Multiview) which allows one-click cropping of a live 3D image (Figure 8).

* It permits the detection of very small or tiny raphe often undetectable by 2D echo due to the fact raphe is not exactly at the plane than non-fused cusp (Figure 9) (10).

\section{Echo protocol: aorta phenotypes and valve analysis (Figure 10)}

The current protocol, product of an elaborate joint effort of the Heart Team members from our institution, is the result of a systematic and standardized 2D/3D TEE assessment of the $\mathrm{AV}$ and ascending aorta.

(I) It starts with the definition of the aortic phenotype, which relies on the measurement of the aortic annulus, the sinuses of Valsalva, the sinotubular 
LCC

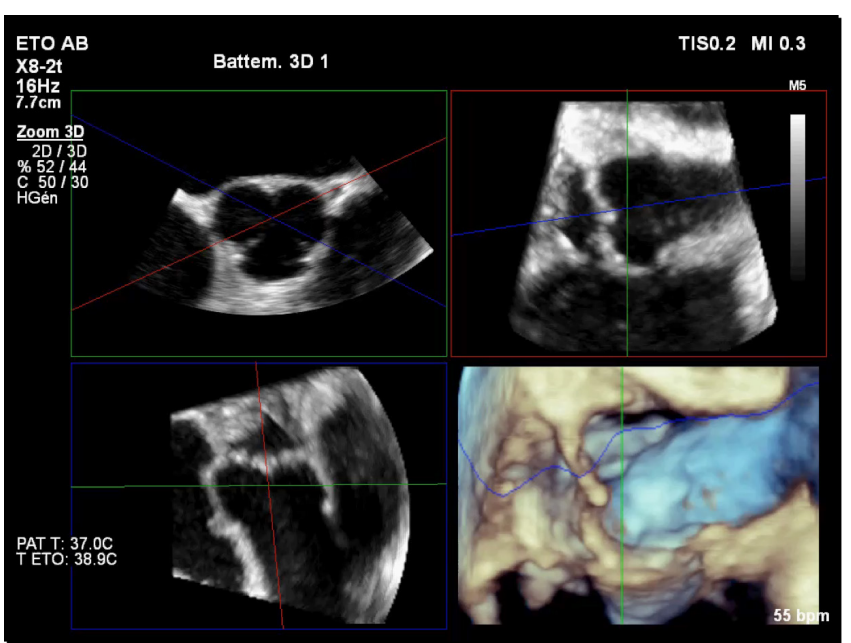

NCC

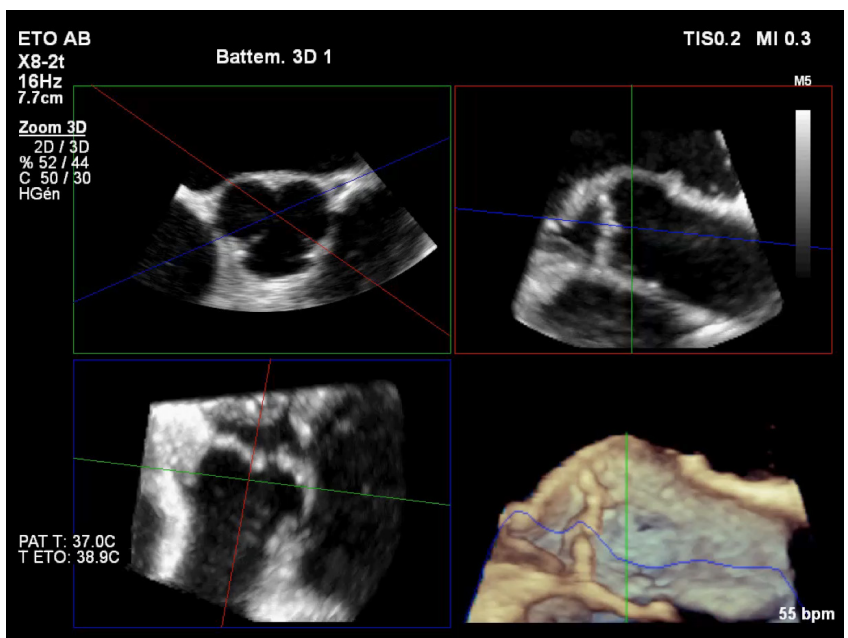

Figure $8 \mathrm{MPR}$ alignment with a live processing tool (Multiview).
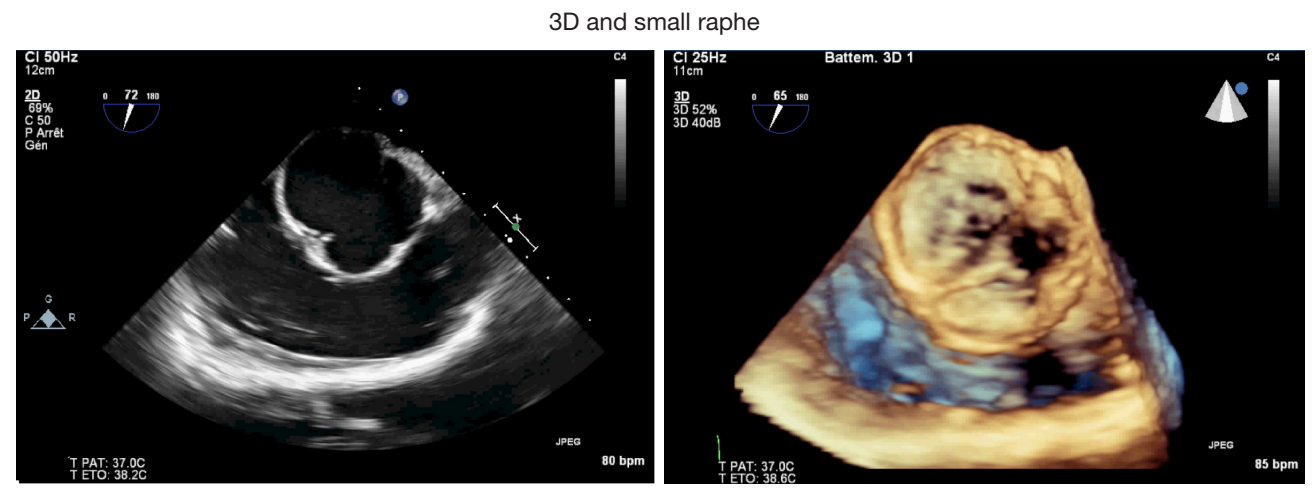

Figure 9 Detection of small raphe with 3D echocardiography.

junction and the ascending aorta, performed by TEE or, alternatively, by ECG gated cardiac CT;

(II) The etiology of the AV disease is based on the distinction between a bicuspid and a tricuspid valvular phenotype;

(III) The dysfunction relies on the analysis of cusp motion and jet characteristics;

(IV) The lesion refers to the quality and quantity of valve tissue;

(V) The impact of the protocol on the feasibility and AV repair strategy.

The aortic phenotype may fall into three categories:

* Normal root and ascending aorta—aortic diameters $<40 \mathrm{~mm}$ : isolated AR;

* Aortic root dilatation-aorta at the sinuses of
Valsalva $>45 \mathrm{~mm}$;

* Ascending aortic aneurysm-aortic root $<40 \mathrm{~mm}$, ascending aorta $>45 \mathrm{~mm}$.

Different methods of aortic measurements have been reported and this may result in diameter discrepancies of $2-3 \mathrm{~mm}$ that could influence therapeutic management. To improve reproducibility, it is recommended to measure diameters using the inner-inner edge technique at end diastole on the strictly transverse plane by double oblique reconstruction perpendicular to the axis of blood flow of the corresponding segment. Diameters at the annulus, sinus of Valsalva, sinotubular junction, tubular ascending aorta and aortic arch level should be reported. Maximum root diameter should be taken from sinus to sinus rather than sinus to commissure diameter, as it correlates more closely 


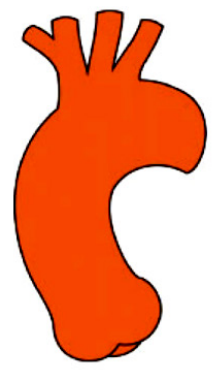

Normal aorta

Type I

Normal cusp movements related to aortic root or ascending aorta dilation with central jet
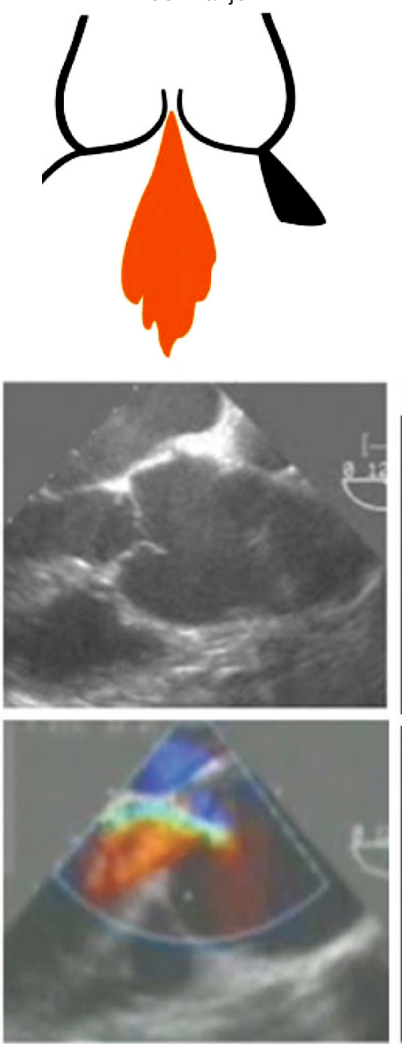

Phenotypes of Aortic Root and Ascending Aorta

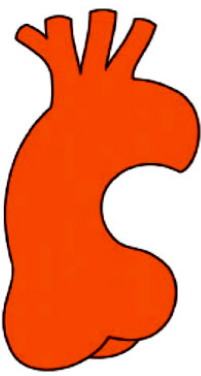

Aortic root dilation

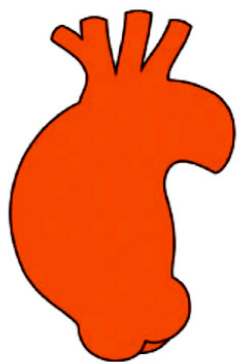

Ascending aorta dilation

\section{Mechanisms of Al Classification}

Type II

Cusp prolapse with eccentric jet
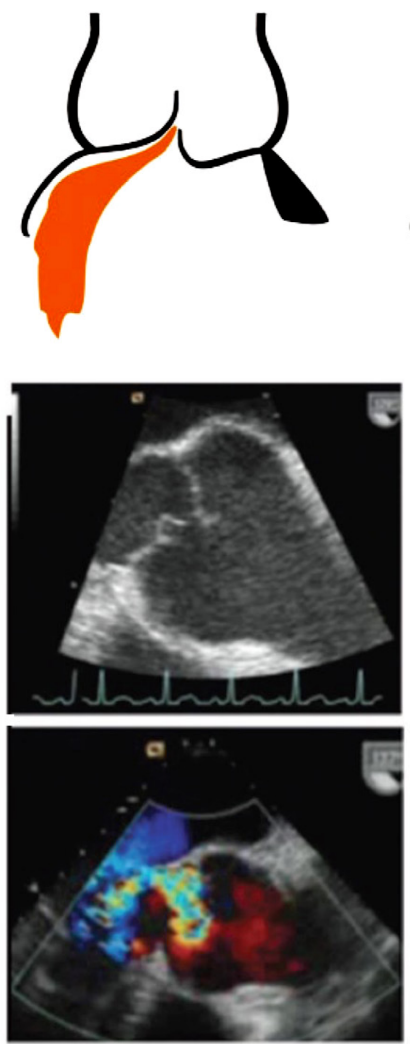

Type III

Cusp retraction with poor tissue quality or quantity with large central
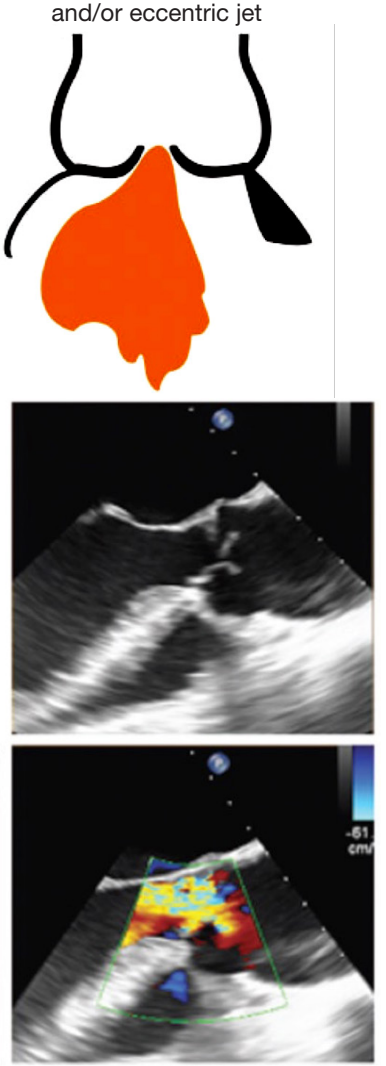

Figure 10 Phenotypes of the proximal aorta associated with the classification of aortic insufficiency mechanisms. Annulus and sinotubular junction dilatation can be associated with any aortic phenotypes as a combined mechanism of aortic insufficiency.

to long-axis leading edge to echo maximum diameters.

Etiology relies on the number of cusps and functional commissures [computational fluid dynamics (CFD) may help with the diagnosis] and the presence/absence of one or several raphes. According to Sievers classification, a bicuspid AV can be subdivided into a type 0 (two functional commissures and no raphe) or a type 1 (two functional commissures and one raphe, most frequently involving the right and the left coronary cusps), whereas an unicuspid valve is defined by the presence of two raphes 


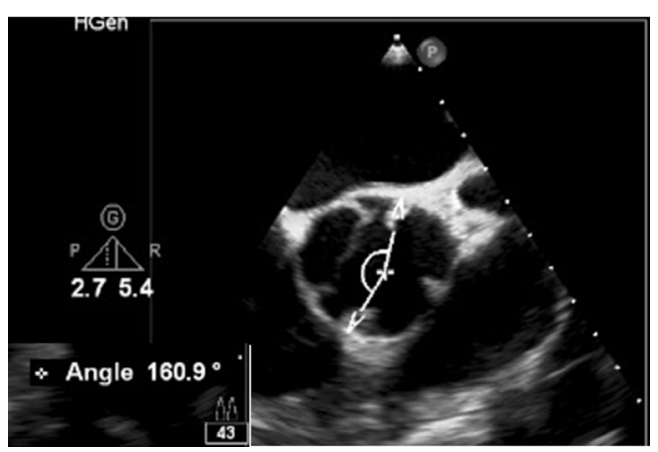

Figure 11 Commissural orientation in bicuspid valve in short axis view.

and one functional commissure between the left and the noncoronary cusp (Figure 1).

In case of a BAV, we need to determine the commissural orientation in diastole (Figure 11), depending on which, the bicuspid valve can be defined as either symmetrical (when the angle between the functional commissures and the center of the non-fused cusp is between $160^{\circ}$ and $180^{\circ}$ ) or asymmetrical (when the angle is $<160^{\circ}$ ), with surgical implications as a need for symmetrization of functional commissures.

The assessment of valve dysfunction is based on cusp motion and jet characteristics. Depending on cusp mobility, there are three types of valve dysfunction: type I characterized by normal cusp motion, associated with root/ascending aorta dilatation (central jet), type II defined as cusp prolapse (eccentric jet) and type III characterized by cusp restriction, associated with poor tissue quality and or quantity (central or eccentric jet). As far as type II dysfunction is concerned, cusp prolapse can be either incomplete (relative), when the cusp free margin lies above the aortic annulus plane, or complete, when the cusp free margin overrides the annulus plane. Again, 3D imaging helps with the differential diagnosis. In case of a complete prolapse, the $\mathrm{eH}$ of the involved cusp is negative, as its free edge falls below the annulus plane in diastole, whereas in case of a relative prolapse, the eH, although reduced, remains positive. Regarding the regurgitant jet, the X Plane CFD assessment offers a simultaneous evaluation of its three attributes (jet origin and extension in the short axis view and jet direction in the long axis view).

The lesion, defined as valve tissue quantity and quality, dictates the feasibility of valve repair. Restriction per se is not considered an absolute contraindication for valve repair, yet when combined with valve retraction it is a predictor of a poor repair result. Echocardiography is employed once more to define the surgical cutoffs (for tricuspid AV, retraction is defined by a surgical $\mathrm{gH}$ of less than $16 \mathrm{~mm}$, whereas for a bicuspid valve, a surgical $\mathrm{gH}$ of the non-fused cusp of less than $19 \mathrm{~mm}$ )

Another lesion with surgical implications is the presence of fenestrations, which appear as filament-like structures on the free margins of the aortic cusps, attached to the commissures, and which require surgical repair if fragile or ruptured. It represents a factor of complexity of repair if these fenestrations are multiple and fragile.

Finally, the valve analysis protocol enables the surgical decision-making process (Table 1):

- Valve retraction (a $\mathrm{gH}<16 \mathrm{~mm}$ in a tricuspid AV or $<19 \mathrm{~mm}$ in a bicuspid valve) contraindicates the repair;

* Preoperative eH measurement helps with the decision of cusp resuspension (if $<9 \mathrm{~mm}$ ); post repair, it serves to assess the symmetry of the repair;

- A coaptation length $\geq 5$ mm post AV repair predicts a more durable result;

* A preoperative commissure orientation $<160^{\circ}$ in a bicuspid AV indicates the need for restoring cusp symmetry;

* Fenestrations, if multiple and fragile, may hinder valve repair.

\section{What is a satisfying result after $\mathbf{A V}$ repair?}

Systematic evaluation of AV repair by immediate postoperative TEE (including 3D-imaging) performed in the operating room is mandatory. The ideal result is obviously the absence of any residual AV regurgitation, without cusp restriction and without significant transvalvular gradient. If a residual valvular leak is present, the coaptation length between the cusps is an important predictor of long-term outcome (11). The retrospective analysis of 186 consecutive patients who underwent AV repair during a 10 -year period showed that a coaptation length $\geq 4 \mathrm{~mm}$ was associated with minimal recurrence of moderate/severe AR after 4 years, even in the presence of mild early postoperative AR. In contrast, a coaptation length $<4 \mathrm{~mm}$ or a coaptation line below the aortic annulus were associated with a significant rate of recurrent $A R$ at mid-term.

The second important determinant of mid-term AR recurrence is early postoperative eH (12). Based on a series of 316 patients who underwent reconstruction of a regurgitant bicuspid $\mathrm{AV}$, the authors showed that a 


\begin{tabular}{|c|c|c|c|c|c|}
\hline Measurement & Echo definition & Echo views \& modalities & $\begin{array}{l}\text { Cut-off } \\
\text { value }\end{array}$ & Echo significance & Surgical implications \\
\hline $\begin{array}{l}\text { Aortic } \\
\text { annulus }\end{array}$ & $\begin{array}{l}\text { Distance between hinge of } \\
\text { right cusp and commissure } \\
\text { L/N (end-systole) }\end{array}$ & Long axis view & $25 \mathrm{~mm}$ & $\begin{array}{l}\text { If } \geq 25 \mathrm{~mm} \text {; dilated } \\
\text { annulus }\end{array}$ & $\begin{array}{l}\text { Need for aortic } \\
\text { annuloplasty } \\
\text { techniques (ring) }\end{array}$ \\
\hline $\begin{array}{l}\text { Effective } \\
\text { height }(\mathrm{eH})\end{array}$ & $\begin{array}{l}\text { Distance between free } \\
\text { edge of individual cusp } \\
\text { and plane of the annulus } \\
\text { (end-diastole) }\end{array}$ & $\begin{array}{l}\text { Long axis or deep } \\
\text { transgastric view. X Plane for } \\
\text { RCC; Live MPR Multiview or } \\
\text { Q Lab post-processing }\end{array}$ & $9 \mathrm{~mm}$ & $\begin{array}{l}\text { Prolapse definition: } \\
\text { eH }<0 \text { complete; } \\
0<\mathrm{eH}<9 \mathrm{~mm} \\
\text { incomplete }\end{array}$ & $\begin{array}{l}\text { Pre-op: need for } \\
\text { resuspension; post- } \\
\text { pump: criteria of } \\
\text { effective and durable } \\
\text { repair }\end{array}$ \\
\hline $\begin{array}{l}\text { Geometric } \\
\text { height }(\mathrm{gH})\end{array}$ & $\begin{array}{l}\text { Length of individual cusp } \\
\text { (origin insertion hinge to } \\
\text { free edge, in end-diastole) }\end{array}$ & $\begin{array}{l}\text { Long axis or deep } \\
\text { transgastric view. X Plane for } \\
\text { RCC; Live MPR Multiview or } \\
\text { Q Lab post-processing }\end{array}$ & $\begin{array}{l}16 \mathrm{~mm} \text { in } \\
\text { tricuspid; } \\
19 \mathrm{~mm} \text { in } \\
\text { bicuspid }\end{array}$ & $\begin{array}{l}\text { Cusp retraction if: } \\
<16 \mathrm{~mm} \text { (tricuspid); } \\
<19 \mathrm{~mm} \text { (bicuspid) } \\
\text { "surgical cut-off" }\end{array}$ & $\begin{array}{l}\text { Unfavorable lesions for } \\
\text { aortic valve repair }\end{array}$ \\
\hline $\begin{array}{l}\text { Coaptation } \\
\text { height }(\mathrm{cH})\end{array}$ & $\begin{array}{l}\text { Distance of coaptation } \\
\text { between two cusps (end- } \\
\text { diastole) }\end{array}$ & $\begin{array}{l}\text { Long axis or deep } \\
\text { transgastric view }\end{array}$ & $5 \mathrm{~mm}$ & $\begin{array}{l}\text { Good coaptation if } \\
>5 \mathrm{~mm}\end{array}$ & $\begin{array}{l}\text { Post-pump: criteria of } \\
\text { effective and durable } \\
\text { repair }\end{array}$ \\
\hline $\begin{array}{l}\text { Commissural } \\
\text { orientation }\end{array}$ & $\begin{array}{l}\text { Angle between two } \\
\text { functional commissures in } \\
\text { type I bicuspid valve (end- } \\
\text { diastole) }\end{array}$ & $\begin{array}{l}\text { Short axis view; alternative: } \\
\text { CT }\end{array}$ & $160^{\circ}$ & $\begin{array}{l}\text { If }<160^{\circ} \\
\text { asymmetric } \\
\text { configuration of } \\
\text { bicuspid type I }\end{array}$ & $\begin{array}{l}\text { Need for } \\
\text { symmetrization } \\
\text { of functional } \\
\text { commissures }\end{array}$ \\
\hline
\end{tabular}

L/N, left/non; RCC, right coronary cusp.

postoperative $\mathrm{eH} \geq 9 \mathrm{~mm}$ was associated with very few $\mathrm{AV}$ reoperations after 10 years, as opposed to a reoperation rate close to $50 \%$ in case of eH $<9 \mathrm{~mm}$.

In summary, based on 3D-TEE performed in the OR, the result of $\mathrm{AV}$ repair is satisfying in cases of:

* No residual AR or minimal central AR jet (trace);

* Individual cusps eH $\geq 9 \mathrm{~mm}$;

* Inter-cusp coaptation length $\geq 4 \mathrm{~mm}$;

* Aortic annulus (basal virtual plane/systole) <25 mm;

* No cusp restriction and mean transaortic pressure gradient $<10 \mathrm{mmHg}$.

\section{When AV repair is not optimal: when to reclamp?}

The presence of more than trivial $\mathrm{AR}$ after repair defines a suboptimal result. In this case, the AR jet direction is crucial for subsequent surgical management.

\section{Case \#1: residual AR with an eccentric jet}

Most instances of residual AR (more than trace) with an eccentric jet are an indication to reclamp for a second AV repair, or valve replacement. Eccentric AR may be due to residual cusp prolapse (typically with a "stair step" aspect, Figure 12), which may be treated by repeat cusp resuspension.

On the other hand, eccentric AR might be due to cusp restriction, to be treated by the release of some resuspension stitches if possible, or by AV replacement. The management of residual eccentric AR is summarized in Figure 13.

\section{Case \#2: residual AR with a central jet (Figure 14)}

A residual central AR is acceptable if it's not more than trivial, meaning:

* Limited extension of the color Doppler jet in the LV outflow tract, far from the free edge of the anterior mitral leaflet;

* Vena contracta of the jet $<2 \mathrm{~mm}$;

- Effective regurgitant area less than $10 \mathrm{~mm}^{2}$.

Otherwise, the possible causes of significant (grade $\geq 1 / 4$ ) residual central AR might be:

* An oversized aortic annulus (>25 mm of diameter in systole), that can be treated by undersized annuloplasty, performed at beating heart;

* A symmetric restriction of the aortic cusps, that can 


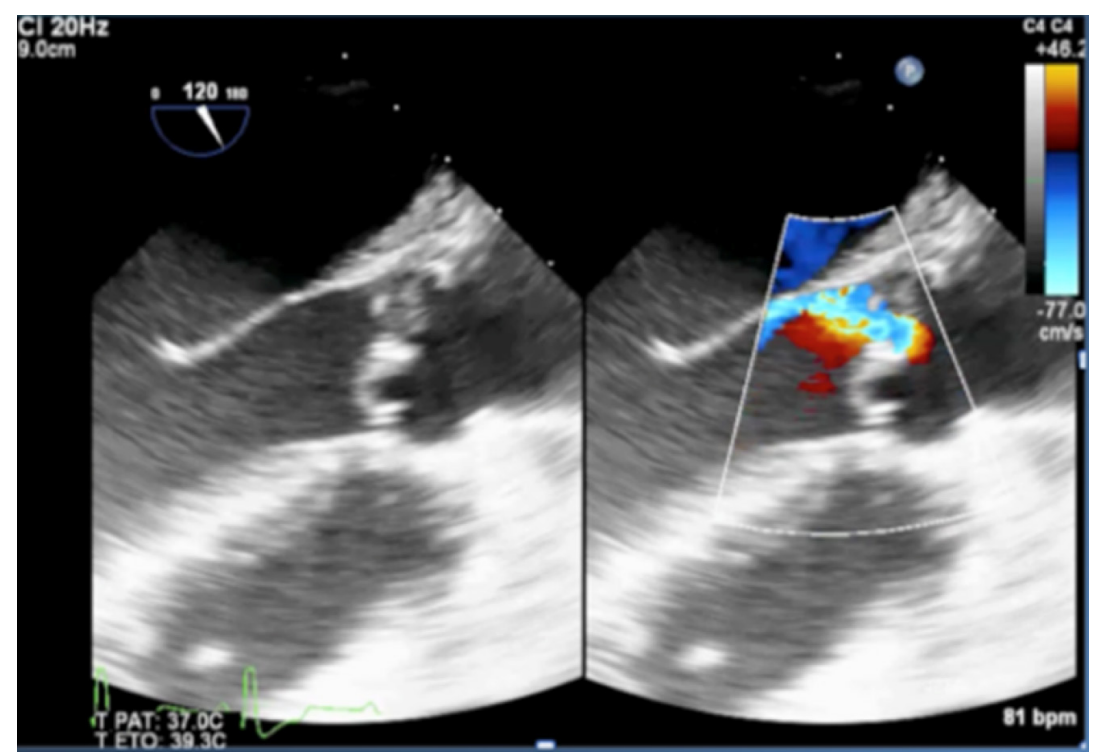

Figure 12 Residual aortic regurgitation with "stair step" aspect, eccentric jet and right cusp prolapse.

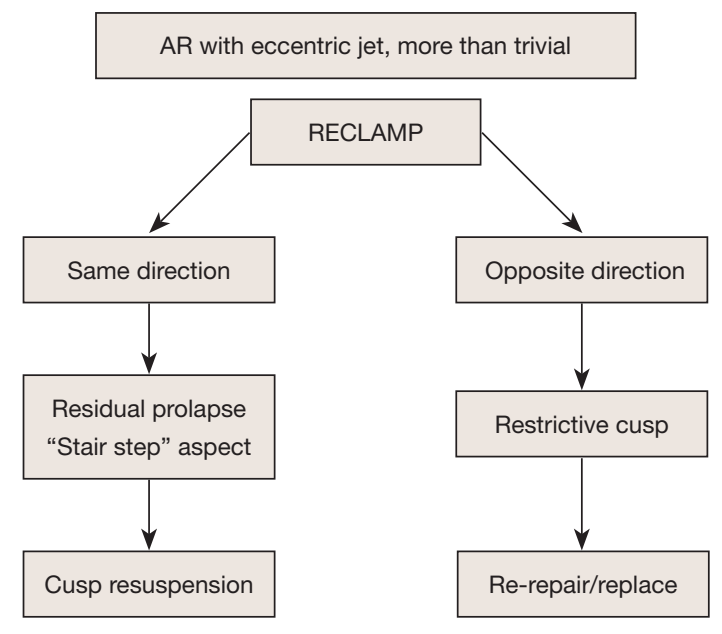

Figure 13 Algorithm 1: management of residual eccentric AR. AR, aortic regurgitation.

necessitate AV replacement.

\section{Conclusions}

AV repair is feasible in all patients with either bi or tricuspid valves, regardless of the aortic phenotype and type of dysfunction, in the absence of valve retraction. In general, better durability of AV repair is observed in patients with type 1 and $2 \mathrm{AR}$ compared to those with type 3. Currently, we would not recommend AV repair

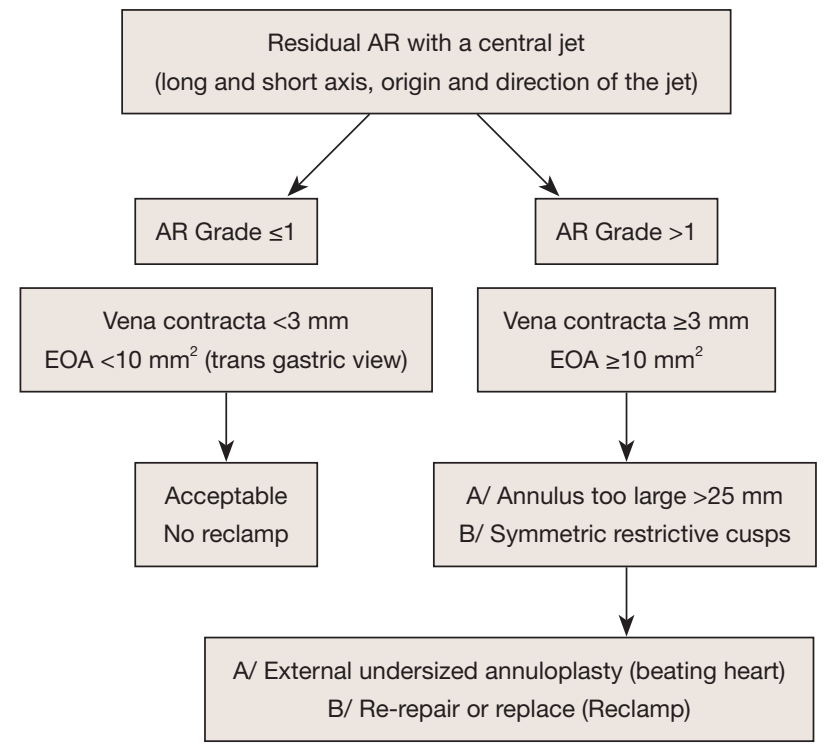

Figure 14 Algorithm 2: management of residual central AR. AR, aortic regurgitation.

for a patient with a pure type $3 \mathrm{AR}$ mechanism except in selected pediatric or adolescent patients. However, we would still consider a type 3 repair in patients in whom the retraction or calcification is limited to a valve where the main mechanism of $\mathrm{AI}$ is a type 1 or 2 (i.e., BAV with a fibrous or calcified raphe). Furthermore, indications for AV repair must be balanced with age, complexity of the lesion 
and left ventricular function. This protocol is critical to an enhanced team approach by providing a common language between surgeons and echocardiographists, increasing the number of AV repairs for selected patients in expert centers.

\section{Acknowledgments}

None.

\section{Footnote}

Conflicts of Interest: The authors have no conflicts of interest to declare.

\section{References}

1. Baumgartner H, Falk V, Bax JJ, et al. 2017 ESC/EACTS Guidelines for the management of valvular heart disease. Eur Heart J 2017;38:2739-91.

2. Iung B, Baron G, Butchart EG, et al. A prospective survey of patients with valvular heart disease in Europe: The Euro Heart Survey on Valvular Heart Disease. Eur Heart J 2003;24:1231-43.

3. Lansac E, de Kerchove L. Aortic valve repair techniques: state of the art. Eur J Cardiothorac Surg 2018;53:1101-7.

4. Vanoverschelde JL, van Dyck M, Gerber B, et al. The role of echocardiography in aortic valve repair. Ann Cardiothorac Surg 2013;2:65-72.

Cite this article as: Berrebi A, Monin JL, Lansac E. Systematic echocardiographic assessment of aortic regurgitationwhat should the surgeon know for aortic valve repair? Ann Cardiothorac Surg 2019;8(3):331-341. doi: 10.21037/ acs.2019.05.15
5. Van Dyck MJ, Watremez C, Boodhwani M, et al. Transesophageal echocardiographic evaluation during aortic valve repair surgery. Anesth Analg 2010;111:59-70.

6. Sievers HH, Schmidtke C. A classification system for the bicuspid aortic valve from 304 surgical specimens. J Thorac Cardiovasc Surg 2007;133:1226-33.

7. Bierbach BO, Aicher D, Issa OA, et al. Aortic root and cusp configuration determine aortic valve function. Eur J Cardiothorac Surg 2010;38:400-6.

8. Schäfers HJ, Schmied W, Marom G, et al. Cusp height in aortic valves. J Thorac Cardiovasc Surg 2013;146:269-74.

9. Hagendorff A, Evangelista A, Fehske W, et al. Improvement in the Assessment of Aortic Valve and Aortic Aneurysm Repair by 3-Dimensional Echocardiography. JACC Cardiovasc Imaging 2019. [Epub ahead of print].

10. Sperling JS, Lubat E. Forme fruste or 'Incomplete' bicuspid aortic valves with very small raphes: The prevalence of bicuspid valve and its significance may be underestimated. Int J Cardiol 2015;184:1-5.

11. le Polain de Waroux JB, Pouleur AC, Robert A, et al. Mechanisms of recurrent aortic regurgitation after aortic valve repair: predictive value of intraoperative transesophageal echocardiography. JACC Cardiovasc Imaging 2009;2:931-9.

12. Aicher $\mathrm{D}$, Kunihara $\mathrm{T}$, Abou Issa $\mathrm{O}$, et al. Valve configuration determines long-term results after repair of the bicuspid aortic valve. Circulation 2011;123:178-85. 